\title{
ENDEMIC FISHES OF THE CORTEZ BIOGEOGRAPHIC PROVINCE (EASTERN PACIFIC OCEAN)
}

\author{
Deivis S. PALACIOS-SALGADO ${ }^{1,2^{*}}$, Luis A. BURNES-ROMO ${ }^{2,3}$, José J. TAVERA ${ }^{4}$, \\ and Arturo RAMÍREZ-VALDEZ ${ }^{5,6}$
}

${ }^{1}$ Escuela Nacional de Ingeniería Pesquera, Universidad Autónoma de Nayarit, Bahía de Matanchén, San Blas Nayarit, México

2 IIK'KAKNAB A.C. Bahía Asunción 181, Col. Fovissste, 23060, La Paz, Baja California Sur, Mexico

${ }^{3}$ Secretaria de Agricultura, Ganadería, Desarrollo Rural, Pesca y Alimentación (SAGARPA).

${ }^{4}$ Centro de Investigaciones Biológicas del Noroeste, S.C. (CIBNOR), Mexico

${ }^{5}$ Facultad de Ciencias Marinas, Universidad Autónoma de Baja California (UABC),

Ensenada, Baja California, Mexico

${ }^{6}$ Instituto de Investigaciones Oceanológicas (IIO), UABC, Ensenada, Baja California, Mexico

Palacios-Salgado D.S., Burnes-Romo L.A., Tavera J.J., Ramírez-Valdez A. 2012. Endemic fishes of the Cortez Biogeographic Province (eastern Pacific Ocean). Acta Ichthyol. Piscat. 42 (3): 153-164.

\begin{abstract}
Background. The Cortez Province (CP) is located in the transitional warm-temperate/subtropical region that allows the ichthyological component inhabiting it to be a mixture of elements of different biogeographic affinities. Since the first systematic analysis of the fish fauna of the Gulf of California in the 1960's a major portion of the endemic species was recognized. Subsequently, a total of 31 new endemic species have been described in the CP. This study constitutes an amendment of the fish component of the CP, including the most relevant ecological attributes of the species, along with an updated taxonomic list.

Materials and methods. A comprehensive literature review was made, considering current biological knowledge, and taxonomic status of the endemic species from the CP. Those fish species with restricted distribution ranges, falling within the limits of this province, were considered endemic. Additionally, and to recognize the dominant ecological attributes of the $\mathrm{CP}$ endemic species, the preferential habitat, bathymetric distribution, the reproduction strategy, and the maximum total length (TL) were recorded.

Results. Seventy-nine endemic species were recognized and grouped in 13 orders, 29 families, and 59 genera. Gobiidae (12), Chaenopsidae (8), and Labrisomidae (7) are the families with the highest species richness, and Sebastes (6 species), the most diverse genus. Forty-five percent of the species are associated with coral and rocky reefs, with $35 \%$ distributed within the first $10 \mathrm{~m}$ depth layer. The dominant reproduction strategies are: oviparous with benthic eggs and pelagic larval phase (48.7\%), and oviparous with pelagic eggs (25.6\%). More than half of the species (52\%) are shorter than $10 \mathrm{~cm}$ (total length).

Conclusion. The list of endemic species presented in this study is not conclusive, still undescribed species have not been included, detected differences (morphologic and/or genetic) in several species with disjunct populations may increase the diversity of this province.
\end{abstract}

Keywords: Gulf of California, endemic species, biogeography, Gobiidae

\section{INTRODUCTION}

The Gulf of California (GC) represents one of the world's most productive sea areas including an important diversity of marine environments (e.g., rocky- and coraline reefs, oceanic trenches, lagoons, and wetlands) (Thomson et al. 2000, Thomson and Gilligan 2002, Brusca et al. 2005). The GC oceanographic conditions, geographic location, and geological history have deeply influenced speciation processes and species accumulation, which may explain why this area includes the second highest peak in species richness within the Tropical Eastern Pacific (TEP) (Mora and Robertson 2005, Robertson and Allen, 2008). Its location in the transitional warm-temperate and subtropical biogeographic region allows the fishes inhabiting to be a mixture of elements from different affinities: tropical, subtropical, temperate,

\footnotetext{
* Correspondence: Dr. Deivis S. Palacios Salgado, Escuela Nacional de Ingeniería Pesquera, Colección ictiológica, Apartado Postal 10, San Blas, Nayarit. México 63740, phone: (+323) 231-21-20, e-mail: palaciossalgado@gmail.com.
} 
and arctic-boreal or warm-temperate (Walker 1960, Castro-Aguirre et al. 1995, Hastings et al. 2010).

Given the high number of reported endemisms from different taxonomic groups, Briggs (1974) recognized the inner sea of the Baja California Peninsula as a biogeographic province different from the San Diegan and Mexican Provinces, naming it the Cortez Province (CP). The latter author determined the southern limits to be at La Paz Bay on the west coast of the Gulf of California, and Topolobampo Bay on the east coast. This province together with the San Diegan Province constitutes the warm-temperate Californian region. Currently, the northern limit of the CP is known to be located at Bahía Magdalena, Baja California Sur, and the southern boundary in the Topolobampo region, Sinaloa (Fig. 1) (Hastings 2000, Robertson and Cramer 2009). This province is delimited to the north by strong temperature gradients (Robertson et al. 2004, Mora and Robertson 2005) and to the south by the Sinaloa gap, a coastline of $370 \mathrm{~km}$, which includes wide extensions of sandy and muddy bottoms, with estuarine lagoons and wide mangrove areas that separate it from the Mexican Province (Springer 1959, Dawson 1975, Hastings 2000). This gap has been considered a 'faunistic filter' given that it does not have the same isolation effects for all fish elements (Castro-Aguirre et al. 1995).

Walker (1960) made the first evaluation of the endemic fishes of the Gulf of California reporting 92 species. In subsequent studies, Findley et al. (1996a, b, 1997, 1999) initially recognized the existence of 77 , and ended up with 86 , endemic species. Castro-Aguirre et al. (2005a) evaluated fish species from the Gulf of California with amphipacific, boreal, endemic, and amphipeninsular distributions and reported 50 endemic species. Nevertheless, despite these studies mention endemic species of the GC, none of them present a complete systematic list. Our study presents an amendment of the fish component from the $\mathrm{CP}$, including the most relevant ecological attributes of species, plus an updated taxonomic list.

\section{MATERIAL AND METHODS}

A comprehensive literature review was made, considering current biological knowledge and taxonomic status of the endemic species from the Cortez Province (CP) (sensu Hastings 2000). Those fish species with restricted distribution ranges, falling within the limits of this province, were considered endemic. An updated systematic list is presented excluding species being a subject of any stage of taxonomic review. Family designations and higher hierarchical ranks follow Nelson (2006). Genera and their respective species are presented alphabetically. The spelling of scientific and common names is based on FishBase (Froese and Pauly 2012).

Additionally, and to recognize the dominant ecological attributes of $\mathrm{CP}$ endemic species, the preferential habitat, bathymetric distribution, reproduction strategy, and the maximum total length (TL) were recorded. According to their habitat, species were classified as follows: reef species (R); soft bottom demersal (SBD); mixed bottom demersal (MBD); pelagic-demersal (PD), species that being demersal also break-into the water column; neriticpelagic (NP), those species associated to the upper part of the water column by the coastal zone; mesopelagic (MP); and bathybenthic (BB). Classification in bathymetric distribution was made using minimum and maximum depth limits in which species are distributed.

Reproductive strategies for each species were grouped according to Balon (1989) and Elliot and Dewailly (1995), as following: viviparous (V), those species who give birth to complete juveniles and whose embryos obtain nutrients from yolk or directly from their mother; ovoviviparous (W), those with internal fertilization, embryonic development is produced inside the ovary until larvae are formed, and nutrition of embryo does not depend on the mother but on egg yolk; and oviparous, in those fishes, eggs are spawned directly to the environment and fertilization is external although in some uncommon instances internal fertilization events may occur before spawning. Within the oviparous group, a sub-classification was considered: oviparous with pelagic eggs (OP), oviparous with benthic eggs and pelagic phase (OBPP), oviparous with benthic eggs without pelagic phase (OBWPP), oviparous with oral gestation $(\mathrm{OOG})$, and oviparous with gestation in the vascularized ventral sac (OGVVS).

Regarding size, species were classified by $10 \mathrm{~cm}$ length intervals. Biological information was obtained from specific published sources (e.g., Thomson et al. 2000, Robertson and Allen 2008).

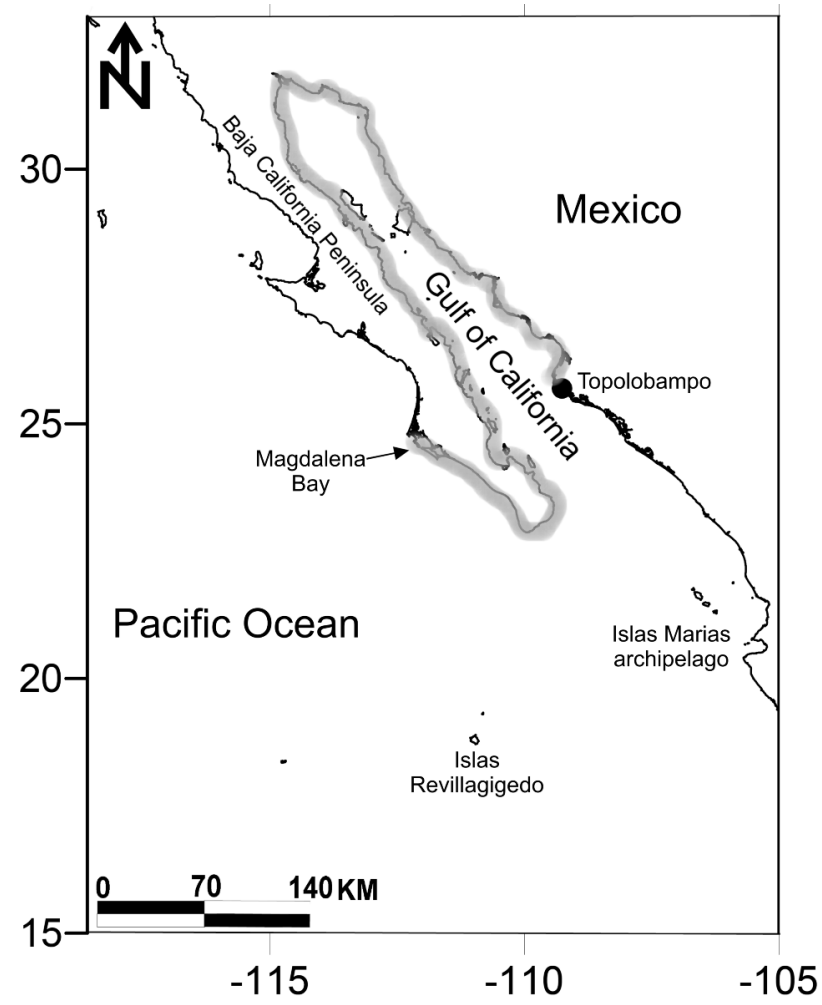

Fig. 1. Study area, the biogeographic province of Cortez (PC) (shaded grey) (sensu Hastings 2000) 


\section{RESULTS}

Seventy-nine endemic species were recognized and grouped into 29 families, and 59 genera (Table 1). A total of 75 species belong to the class Actinopterygii (94.9\%), three to the class Chondrichthyes (3.8\%), and one to the class Myxini (1.3\%). Gobiidae, Chaenopsidae, and Labrisomidae were the best represented families in terms of number of species with 12,8 , and 7 , respectively. On the other hand, Sebastes and Ogilbia (with six and four species, respectively) were the genera with the highest richness. From the overall taxonomic composition, five genera appeared to be endemic to the CP (Colpichthys, Totoaba, Crocodilichthys, Xenomedea, and Aruma).

Fishes associated to coralline- and rocky reef systems were the dominant group with 36 species (45.6\%). Nineteen $(24.05 \%)$ species were grouped in the soft bottom demersal group; the demersal-pelagic and demersalneritic groups were represented by eight species each (Fig. 2). Twenty-seven species $(35.5 \%)$ can be found within the first 10-m depth layer of water (Fig. 3). Six species reach depths $>200 \mathrm{~m}$, of which, only two exceed $1000 \mathrm{~m}$. The dominant reproduction strategies were oviparous with benthic eggs and pelagic phase (38 species, $48.7 \%$ ), and oviparous species with pelagic eggs (20 species, 25.6\%) (Fig. 4). Regarding adult length, more than half of the endemic species (39 species, 52\%) are between 1 and $10 \mathrm{~cm}$ total length (TL) and only six exceed $50 \mathrm{~cm}$ of TL (Fig. 5).

\section{DISCUSSION}

The 79 endemic species considered in this study constitute $8.67 \%$ of the total fish fauna reported for the Gulf of California (911 species; Hastings et al. 2010) and $6.14 \%$ of the total shore species of the Tropical Eastern Pacific (1285 species) (Robertson and Allen 2008). The number of reported endemic fish species has not drastically changed within the past 50 years, from the 92 species documented by Walker (1960), or the 77-86 found by Findley et al. (1996a,b, 1997, 1999). However, the taxonomic list of species that take part of this endemic assemblage has indeed been modified. Since 1960, a total of 31 endemic new species have been described as species new to science in the CP. Ten of these new species were described after Findley et al. (1996a, b, 1997 and 1999): Elacatinus limbaughi* (Gobiidae); Stellifer wintersteenorum (Sciaenidae); Opistognathus fossoris and O. walkeri (Opistognathidae); Mustelus albipinnis (Triakidae); Ogilbia davidsmithi, O. nigromarginata, O. nudiceps (Bythitidae); Etropus ciadi (Paralichthyidae); and Acanthemblemaria hastingsi (Chaenopsidae).

The greatest percentage of endemic species corresponded to the families Gobiidae (13 species), Chaenopsidae (8 species), Labrisomidae (7 species), Scorpaenidae (6 species), Gobiesocidae (5 species), Sciaenidae (5 species), Bythitidae (4 species), and Dactyloscopidae (3 species). Highly diversified families in the Tropical Eastern Pacific such as Serranidae (56 species), Ophichthidae (41 species), Haemulidae (37 species), Labridae (36 species), Carangidae (35 species), and Muraenidae (33 species), do not have endemic representatives in the $\mathrm{CP}$ and/or are poorly represented. The same pattern can be identified at a genus level; e.g., anchovies (Anchoa: 19 species), tonguefishes (Symphurus: 18 species), wrasses and moray eels (Halichoeres and Gymnothorax, respectively; both with 12 autochthonous species), among others.

Walker (1960) recognized four endemic genera from the CP, while Findley et al. (1997) only found two (Totoaba and Xenomedea). According to the presently reported study, five genera, four of them currently monotypic, are endemic to the CP (Colpichthys, Totoaba, Crocodilichthys, Xenomedea, and Aruma).

From the endemic species, three belong to the family Atherinopsidae: the false grunion, Colpichthys regis, a common species in the Sonora coastal hypersaline (values > pss 50) lagoons; and Colpichthys hubbsi and Leuresthes sardine, both autochthonous to the northern part of the Gulf of California, and restricted to the delta and mouth of the Colorado River, Sonora (Castro-Aguirre and Espinosa-Pérez 2006). The Panamic flashlightfish, Phthanophaneron harveyi, is the only species of the family Anomalopidae present in the Tropical Eastern Pacific and it is endemic to the CP (Thomson et al. 2000).

Some species of this endemic component support or even keep supporting important fishery pressure. The Gulf weakfish, Cynoscion othonopterus, forms reproductive aggregations from February to May; a period during which it is captured in great numbers. In 2009, the Gulf weakfish capture reached a profit of more than 30 million Mexican pesos (Paredes et al. 2010). The Gulf croaker, Micropogonias megalops, represents almost $27 \%$ of the total fish capture in the Upper Gulf of California (AragónNoriega et al. 2009). The totoaba, Totoaba macdonaldi, supported one of the most important fisheries in the region; nevertheless, uncontrolled fisheries and the decrease of Colorado River flow and thus spawning, breeding, and reproduction of this species brought this fishery to collapse (Cisneros-Mata et al. 1995). Currently, the totoaba is included in NOM-059-SEMARNAT-2010 regulation in the category of risk of extinct, and as Critically Endangered by the IUCN red list of threatened Species. According to Berdegue (1955), during the 1950s, totoaba fisheries at San Felipe were composed of 25 shrimp fishing ships, each capturing an average of five to six tons of totoaba weekly.

Many other endemic species are part of multi-specific fisheries (e.g., Diplectrum sciurus, Paralichthys aestuarius, Stellifer wintersteenorum, and Umbrina wintersteeni). In addition, some small reef species, at least four (Malacoctenus hubbsi, Emblemaria hypacanthus, E. walkeri, and Gobiosoma chiquita), are exploited for the aquarium trade (Piña-Espallargas, unpublished**).

The number of endemic species presented in this study may vary given that there are non-validated records, such as the spiny guitarfish, Rhinobatos spinosus, that was described from a $26 \mathrm{~cm}$ total length specimen, and considered

\footnotetext{
${ }^{*}$ Full species names featuring the authority and the year are privided in Tables 1 and 2.

** Piña-Espallargas R. 2005. La pesquería de especies marinas con fines de ornato en México. El parque marino de Loreto, B.C.S., como estudio de caso. MSc Thesis. Centro Interdisciplinario de Ciencias Marinas, Instituto Politécnico Nacional (CICIMAR-IPN). La Paz, México.
} 
Table 1

Taxonomic list of the endemic fishes from Cortez Province (Eastern Pacific Ocean)

\begin{tabular}{llcccc}
\hline Class, family, and species & Common name & Habitat & $\begin{array}{c}\text { Depth } \\
{[\mathrm{m}]}\end{array}$ & $\begin{array}{c}\text { TL } \\
{[\mathrm{cm}]}\end{array}$ & RS \\
\hline CLASS MYXINI & & & & & \\
FAMILY MYXINIDAE & Cortez hagfish & G & 708 & 48 & OBWPP \\
Eptatretus sinus Wisner et McMillan, 1990 &
\end{tabular}

CLASS CHONDRICHTHYES

FAMILY SCYLIORHINIDAE

Galeus piperatus Springer et Wagner, 1966

Peppered catshark $\quad$ F $\quad 400-1330 \quad 30 \quad$ OBWPP

FAMILY TRIAKIDAE

Mustelus albipinnis Castro-Aguirre, Antuna-Mendiola,

González-Acosta et De la Cruz-Agüero, 2005

E $103-281 \quad 118 \quad$ V

FAMILY RHINOBATIDAE

Rhinobatos spinosus Günther, 1870

Spiny guitarfish

B

CLASS ACTINOPTERYGII

FAMILY OPHICHTHIDAE

Apterichtus gymnocelus (Böhlke, 1953)

Ethadophis byrnei Rosenblatt et McCosker, 1970

FAMILY CONGRIDAE

Heteroconger canabus (Cowan et Rosenblatt, 1974)

FAMILY ENGRAULIDAE

Anchoa analis (Miller, 1945)

Anchoa helleri (Hubbs, 1921)

Anchoviella parri (Hildebrand, 1943)

FAMILY OPHIDIIDAE

Ophidion iris Breder, 1936

FAMILY BYTHITIDAE

Ogilbia davidsmithi Möller, Schwarzhans et Nielsen, 2005

Smith's

coralbrotula

Longfin Pacific

anchovy

Heller's anchovy

Mystery anchovy

$\begin{array}{llll}\text { B } & 30 & 24 & \text { OP }\end{array}$

Ogilbia nigromarginata Möller, Schwarzhans et Nielsen, 2005

Ogilbia nudiceps Möller, Schwarzhans et Nielsen, 2005

Darkfinned

coralbrotula

Naked-headed

coralbrotula

Ogilbia ventralis (Gill, 1863)

Gulf cuskeel

B

3

51

OP

FAMILY BATRACHOIDIDAE

Porichthys mimeticus Walker et Rosenblatt, 1988

\section{Mimetic}

midshipman

$\begin{array}{llll}\text { B } & 20 & 80 & \text { OP }\end{array}$

\section{FAMILY ATHERINOPSIDAE}

Colpichthys hubbsi Crabtree, 1989

Colpichthys regis (Jenkins et Evermann, 1889)

Leuresthes sardina (Jenkins et Evermann, 1889)

Delta silverside

False grunion

Gulf grunion

$\begin{array}{cccc}\text { E } & 10 & 14 & \text { OP } \\ \text { E } & 10 & 10 & \text { OP } \\ \text { E } & - & - & \text { OP }\end{array}$

FAMILY ANOMALOPIDAE

Phthanophaneron harveyi (Rosenblatt et Montgomery, 1976) Gulf flashlightfish FAMILY SYNGNATHIDAE

Syngnathus carinatus (Gilbert, 1892)

Cortez pipefish

B

FAMILY SEBASTIDAE

Sebastes cortezi (Beebe et Tee-Van, 1938)

Cortez rockfish

$\begin{array}{llll}\text { F } & 1100 & 25.5 & \text { W }\end{array}$

Sebastes exsul Chen, 1971

Buccaneer rockfish

D $\quad 200 \quad 31 \quad$ W

Sebastes peduncularis Chen, 1975

Gulf rockfish

F $\quad 440-450 \quad 4.7$ AJ W


Table 1 (cont.)

\begin{tabular}{l} 
Class, family, and species \\
\hline Sebastes sinensis (Gilbert, 1890) \\
Sebastes spinorbis Chen, 1975 \\
Sebastes varispinis Chen, 1975 \\
FAMILY PERISTEDIIDAE \\
Peristedion paucibarbiger Castro-Aguirre \\
et García-Domínguez, 1984
\end{tabular}

et García-Domínguez, 1984

FAMILY SERRANIDAE

Diplectrum sciurus Gilbert, 1892

FAMILY OPISTOGNATHIDAE

Opistognathus fossoris Bussing et Lavenberg, 2003

Opistognathus walkeri Bussing et Lavenberg, 2003

FAMILY SCIAENIDAE

Cynoscion othonopterus Jordan et Gilbert, 1882

Micropogonias megalops (Gilbert, 1890)

Totoaba macdonaldi (Gilbert, 1890)

Stellifer wintersteenorum Chao, 2001

Umbrina wintersteeni Walker et Radford, 1992

FAMILY KYPHOSIDAE

Girella simplicidens Osburn et Nichols, 1916

FAMILY LABRIDAE

Pseudojuloides inornatus (Gilbert, 1890)

FAMILY TRIPTERYGIIDAE

Axoclinus nigricaudus Allen et Robertson, 1991

Crocodilichthys gracilis Allen et Robertson, 1991

FAMILY DACTYLOSCOPIDAE

Dactyloscopus pectoralis Gill, 1861

Gillellus ornatus Gilbert, 1892

Myxodagnus opercularis Gill, 1861

FAMILY LABRISOMIDAE

Cryptotrema seftoni Hubbs, 1954

Malacoctenus gigas Springer, 1959

Malacoctenus hubbsi Springer, 1959

Paraclinus altivelis (Lockington, 1881)

Paraclinus magdalenae Rosenblatt et Parr, 1969

Starksia cremnobates (Gilbert, 1890)

Xenomedea rhodopyga Rosenblatt et Taylor, 1971

FAMILY CHAENOPSIDAE

Acanthemblemaria crockeri Beebe et Tee-Van, 1938

Acanthemblemaria hastingsi Lin et Galland, 2010

Chaenopsis coheni Böhlke, 1957

Coralliozetus micropes (Beebe et Tee-Van, 1938)

Coralliozetus rosenblatti Stephens, 1963

Emblemaria hypacanthus (Jenkins et Evermann, 1889)

Emblemaria walkeri Stephens, 1963

Stathmonotus sinuscalifornici (Chabanaud, 1942)

$\begin{array}{lcccc}\text { Common name } & \text { Habitat } & \begin{array}{c}\text { Depth } \\ {[\mathrm{m}]}\end{array} & \begin{array}{c}\mathrm{TL} \\ {[\mathrm{cm}]}\end{array} & \text { RS } \\ \begin{array}{lcccc}\text { Blackmouth } \\ \text { rockfish }\end{array} & \mathrm{F} & 670 & 15.2 & \mathrm{~W} \\ \begin{array}{l}\text { Spinyeye rockfish } \\ \text { Hidden rockfish }\end{array} & \mathrm{D} & 200 & 34.4 & \mathrm{~W} \\ & \mathrm{~F} & 500 & 5.6 \mathrm{AJ} & \mathrm{W} \\ \text { Cortez searobin } & \mathrm{B} & 60 & 8 & \text { OP } \\ & & & & \\ \text { Gulf squirrelfish } & \mathrm{B} & 100 & 17 & \text { OP } \\ \begin{array}{l}\text { Barred jawfish } \\ \text { C }\end{array} & \mathrm{C} & 90 & 13.5 & \text { OOG }\end{array}$

$\begin{array}{lllll}\text { Gulf weakfish } & \text { D } & 30 & 70 & \text { OP }\end{array}$

$\begin{array}{lllll}\text { Gulf croaker } & \text { D } & 30 & 40 & \text { OP }\end{array}$

$\begin{array}{lllll}\text { Totoaba } & \text { D } & 25 & 200 & \text { OP }\end{array}$

$\begin{array}{lllll}\text { Amigo stardrum } & \text { D } & 20 & 21 & \text { OP }\end{array}$

$\begin{array}{lllll}\text { Wintersteen drum } & \text { D } & 15 & 35 & \text { OP }\end{array}$

$\begin{array}{lllll}\text { Gulf opal eye } & \text { A } & 15 & 46 & \text { OP }\end{array}$

$\begin{array}{lllll}\text { Cape wrasse } & \text { D } & 57 & 9 & \text { OP }\end{array}$

$\begin{array}{lllll}\text { Cortez triplefin } & \text { A } & 5 & 5 & \text { OBPP }\end{array}$

$\begin{array}{lllll}\text { Lizard triplefin } & \text { A } & 40 & 8 & \text { OBPP }\end{array}$

Whitesaddle

stargazer

Ornate stargazer

Dart stargazer

Hidden blenny

Sonora blenny

Redside blenny

Topgallant blenny

Magdalena blenny

Fugitive blenny

Redrump blenny

$\begin{array}{lll}45 & 5 & \text { OBPP } \\ 55 & 6 & \text { OBPP } \\ 20 & 9 & \text { OBPP }\end{array}$

Browncheek blenny

Cortez barnacle

blenny

Cortez pikeblenny

Scarletfin blenny

Spikefin blenny

Gulf signal blenny

Elusive signal

blenny

California worm blenny
A

A

A

A

A

A

A

A

A

A

A

A

A

A

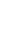

10

3.5

513

69

OBPP

OBPP

$40 \quad 6 \quad$ OBPP

$\begin{array}{lll}21 & 4.5 & \text { OBPP }\end{array}$

$\begin{array}{ll}60 & 4\end{array}$

$\begin{array}{lll}33 & 6.5 \quad \mathrm{~W}\end{array}$


Table 1 (cont.)

\begin{tabular}{|c|c|c|c|c|c|}
\hline Class, family, and species & Common name & Habitat & $\begin{array}{l}\text { Depth } \\
{[\mathrm{m}]}\end{array}$ & $\begin{array}{c}\mathrm{TL} \\
{[\mathrm{cm}]}\end{array}$ & $\mathrm{RS}$ \\
\hline \multicolumn{6}{|l|}{ FAMILY GOBIESOCIDAE } \\
\hline Gobiesox pinniger Gilbert, 1890 & Tadpole clingfish & A & 5 & 13 & OBPP \\
\hline Gobiesox schultzi Briggs, 1951 & Smoothlip clingfish & A & 5 & 7.8 & OBPP \\
\hline Pherallodiscus funebris (Gilbert, 1890) & $\begin{array}{l}\text { Northern fraildisc } \\
\text { clingfish }\end{array}$ & A & 5 & 12.5 & OBPP \\
\hline Tomicodon boehlkei Briggs, 1955 & Cortez clingfish & A & 12 & 7.5 & OBPP \\
\hline Tomicodon humeralis (Gilbert, 1890) & Sonora clingfish & $\mathrm{A}$ & 5 & 10 & OBPP \\
\hline \multicolumn{6}{|l|}{ FAMILY GOBIIDAE } \\
\hline Aruma histrio (Jordan, 1884) & Slow goby & A & 15 & 6.5 & OBPP \\
\hline Barbulifer pantherinus (Pellegrin, 1901) & Panther goby & $\mathrm{A}$ & 32 & 5.2 & OBPP \\
\hline Chriolepis minutillus Gilbert, 1892 & Rubble goby & A & 45 & 3.2 & OBPP \\
\hline Chriolepis zebra Ginsburg, 1938 & Gecko goby & A & 30 & 4.4 & OBPP \\
\hline Elacatinus limbaughi Hoese et Reader, 2001 & $\begin{array}{l}\text { Widebanded } \\
\text { cleaning goby }\end{array}$ & A & 30 & 3.4 & OBPP \\
\hline Evermannia longipinnis (Steindachner, 1879) & Enigmatic goby & $\mathrm{B}$ & 3 & 3.8 & OBPP \\
\hline Gillichthys detrusus Gilbert et Scofield, 1898 & Delta mudsucker & B & 10 & 11.1 & OBPP \\
\hline Gillichthys seta (Ginsburg, 1938) & $\begin{array}{l}\text { Shortjaw } \\
\text { mudsucker }\end{array}$ & A & 2 & 6 & OBPP \\
\hline Gobiosoma chiquita (Jenkins et Evermann, 1889) & Sonora goby & A & 10 & 7.5 & OBPP \\
\hline Ilypnus luculentus (Ginsburg, 1938) & Bright goby & $\mathrm{B}$ & 3 & 5.3 & OBPP \\
\hline Pycnomma semisquamatum Rutter, 1904 & Secret goby & A & 20 & 6.3 & OBPP \\
\hline Quietula guaymasiae Jenkins et Evermann, 1889 & Guaymas goby & $\mathrm{B}$ & 5 & 8.5 & OBPP \\
\hline \multicolumn{6}{|l|}{ FAMILY STROMATEIDAE } \\
\hline Peprilus ovatus Horn, 1970 & Shining butterfish & $\mathrm{E}$ & 27 & 13 & $\mathrm{OP}$ \\
\hline \multicolumn{6}{|l|}{ FAMILY PARALICHTHYIDAE } \\
\hline Etropus ciadi van der Heiden et Plascencia González, 2005 & & $\mathrm{~B}$ & 40 & 11.5 & $\mathrm{OP}$ \\
\hline Paralichthys aestuarius Gilbert et Scofield, 1898 & Cortez flounder & B & 45 & 58 & OP \\
\hline \multicolumn{6}{|l|}{ FAMILY PLEURONECTIDAE } \\
\hline Pleuronichthys ocellatus Starks et Thompson, 1910 & Ocellated turbot & $\mathrm{B}$ & 140 & 24 & OP \\
\hline
\end{tabular}

Common names follow those in FishBase (Froese and Pauly 2012); Habitat: A = reef species, $\mathrm{B}=$ soft bottom demersal, $\mathrm{C}=$ mixed bottom demersal, $\mathrm{D}=$ pelagic-demersal (species that being demersal also break-into the water column), $\mathrm{E}=$ neritic-pelagic (those associated to the upper part of the water column by the coastal zone), $\mathrm{F}=$ mesopelagic, $\mathrm{G}=$ bathybenthic; Depth (minimum and maximum depth limits in which species are distributed); TL = maximum total length, $(\mathrm{AJ}=$ all juveniles); $\mathrm{RS}=$ reproduction strategy $(\mathrm{V}=$ viviparous, $\mathrm{W}=$ ovoviviparous, $\mathrm{OP}=$ oviparous with pelagic eggs, $\mathrm{OBPP}=$ oviparous with benthic eggs and pelagic phase, OBWPP = oviparous with benthic eggs without pelagic phase, OOG = oviparous with oral gestation, OGVVS = oviparous with gestation in the vascularized ventral sac).

as a possible juvenile of a different species by Compagno (2005). However, Castro-Aguirre and Espinosa-Pérez (1996) collected and confirmed the existence of this species off the La Paz Bay. A similar situation is that of the mystery anchovy, Anchoviella parri, described from specimens collected in 1926 on board of the Pawnee research cruise off San Felipe. Based on morphological characters Whitehead et al. (1988) proposed that Anchoviella parri might instead be the Upper Gulf of California Anchoa luci$d a$. Similarly, the Gulf rockfish, Sebastes peduncularis, is only known from two juvenile specimens collected with midwater trawls between the southern end of Tiburon Island and Angel de la Guarda Island, at 440-450 m depths
(Chen 1975). However, given the overlapping characters with the Cortez rockfish, S. cortezi, Chen (1975) himself discussed the option of this species being a junior synonym of the Cortez rockfish or even of any other species of Sebastes (see Chen 1975, Love et al. 2002).

Additionally, three species were described and are currently known only from their holotypes. The ordinary eel, Ethadophis byrnei, was sampled during low tide at Puertecitos, western coast of the Gulf of California. The Cortez searobin, Peristedion paucibarbiger, was collected by bottom trawling at $60 \mathrm{~m}$ depth north of La Paz bay, BCS. Lastly, the cape wrasse, Pseudojuloides inornatus, is known only from a juvenile collected near Cabo San Lucas, BCS. 
A smoothhound shark, Mustelus albipinnis, was described from six captured specimens in 2000, at Puerto Adolfo Lopez Mateos, BCS. The same species was also described, in the same year, under the name $M$. hacat, from 36 collected specimens across the Gulf of California; nevertheless, $M$. albipinnis is considered as the valid name according to priority principle (Anonymous 1999). Considering both descriptions, the distribution range of this species comprises the inner parts of the Gulf of California: from north of Isla Angel de la Guarda, to the south-western coast of the Gulf of California including Santa Cruz and Monserrat Islands, and La Ventana Bay, and the Western coast of BCS on the shelf in front of Bahía Magdalena (Castro-Aguirre et al. 2005b, Perez-Jimenez et al. 2005). This species can be kept as an endemic component of CP; however, according to Perez-Jimenez et al. (2005), it is possible that $M$. albipinnis distribution extends southward to the coasts of Ecuador including Galapagos Islands.

Bussing and Lavenberg (2003), in a review of the genus Opistognathus, concluded that Opistognathus mexicanus Allen et Robertson, 1991, considered endemic to the Gulf of California, was instead a juvenile of $O$. punctatus Peters, 1869, which has a wide distribution across the Tropical Eastern Pacific. An opposite case is that of delta mudsucker, Gillichthys detrusus (Gobiidae), this

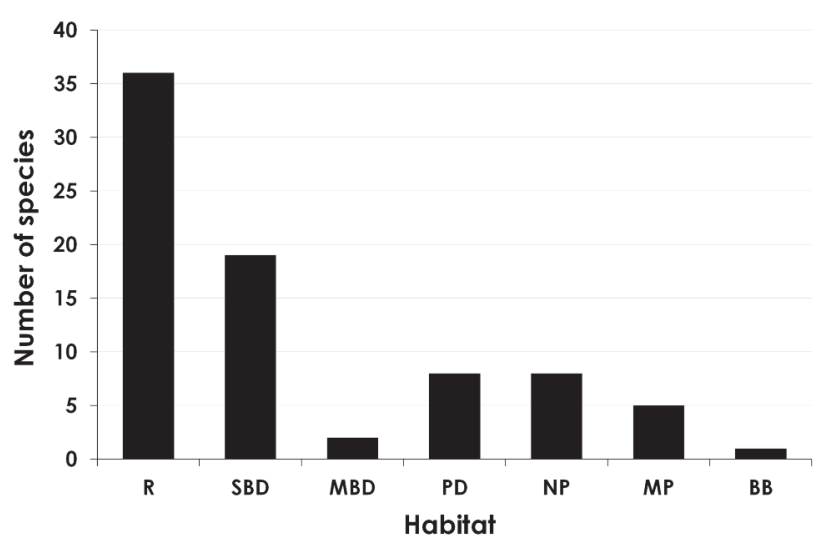

Fig. 2. Endemic fishes from the Cortez Province by preferential habitat; $\mathrm{R}=$ reef, $\mathrm{SBD}=$ soft bottom demersal, $\mathrm{MBD}=$ mixed bottom demersal, $\mathrm{PD}=$ pelagic-demersal, NP = neritic-pelagic, $\mathrm{MP}=$ mesopelagic, $\mathrm{BB}=$ bathybenthic

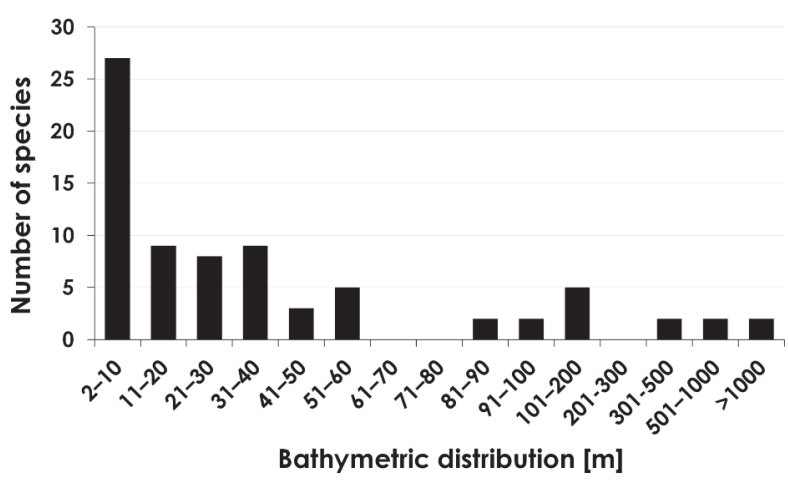

Fig. 3. Bathymetric distribution of endemic fishes from the Cortez Province

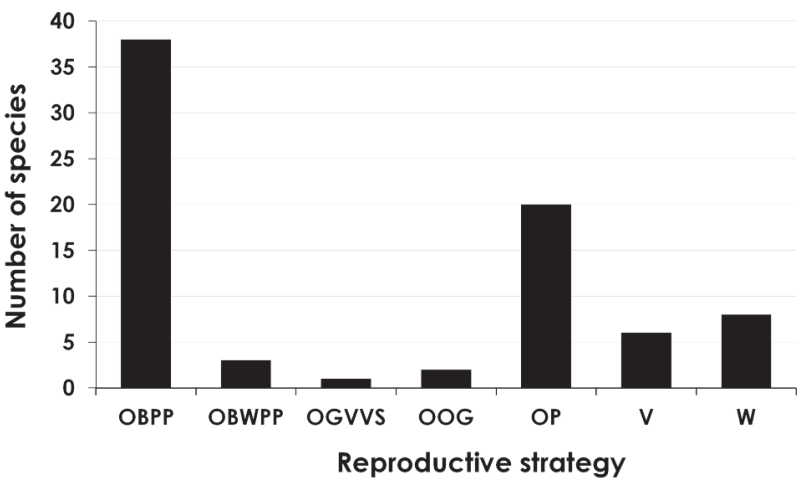

Fig. 4. Reproductive strategies of endemic fishes from the Cortez Province: OBPP = oviparous with benthic eggs and pelagic phase, OBWPP = oviparous with benthic eggs without pelagic phase, OGVVS = oviparous with gestation in the vascularized ventral sac, OOG = oviparous with oral gestation, $\mathrm{OP}=$ oviparous with pelagic eggs, $\mathrm{V}=$ viviparous, $\mathrm{W}=$ ovoviviparous

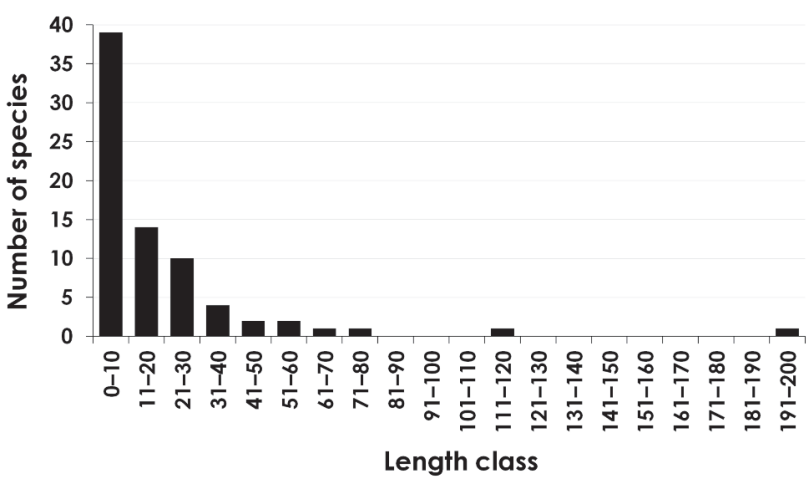

Fig. 5. Endemic fishes from the Cortez Province; Length-class distribution (maximum total length; Length classes [cm]

species was erroneously placed in the synonymy of G. mirabilis Cooper, 1864, in 1907 (see Swift et al. 2011).

A study on several species complexes done by Hastings and Springer (2009), resulted in recognition of existing morphological differences among subspecies of Dactyloscopus pectoralis (fallax, insulatus, and pectoralis) and Malacoctenus hubbsi (hubbsi and polyporosus) evidence used to guarantee at least the existence of $D$. pectoralis and $M$. hubbsi as endemic species to the CP. Similar studies were made by Rosenblatt and Parr (1967, 1969) for the genus Paraclinus; Stepien and Rosenblatt (1991) for Gibbonsia and Heterostichus; and Bath (2008) for the genus Parablennius, where some subspecies were raised to species and some others were recognized as synonyms (Hastings and Springer 2009). Nonetheless, the use of additional tools is required (e.g., geometric morphometrics and genetic or molecular analyses) to come to any further conclusions on these particular cases.

Additionally, 21 species described as endemics of the Gulf of California have been found outside the CP expanding their distribution ranges (e.g., Castro-Aguirre et al. 2006). Those species are: Raja cortezensis 
Genetic divergence in disjunct fish species

\begin{tabular}{|c|c|c|c|}
\hline Species & Molecular marker & $\begin{array}{c}\text { Divergence } \\
{[\%]}\end{array}$ & Reference \\
\hline \multirow[t]{3}{*}{ Anisotremus davidsonii (Steindachner, 1876) } & Cytochrome B & 0.40 & Bernardi and Lape 2005 \\
\hline & Cytochrome B & 1.34 & Bernardi et al. 2003 \\
\hline & S7 & 0 & Bernardi and Lape 2005 \\
\hline Chaenopsis alepidota (Gilbert, 1890) & Control region & 1.87 & Bernardi et al. 2003 \\
\hline Gillichthys mirabilis Cooper, 1864 & Cytochrome B & 2.21 & Huang and Bernardi 2001 \\
\hline \multirow[t]{2}{*}{ Girella nigricans (Ayres, 1860) } & Control region & 8.49 & Bernardi et al. 2003 \\
\hline & Control region & 3.3 & Terry et al. 2000 \\
\hline Gymnura marmorata Cooper, 1864 & NADH2 & $<0.01$ & SCRO \\
\hline Halichoeres semicinctus (Ayres, 1859) & Control region & 0.79 & Bernardi et al. 2003 \\
\hline Hermosilla azurea Jenkins et Evermann, 1889 & Control region & 2.30 & Bernardi et al. 2003 \\
\hline Hypsoblennius jenkinsi (Jordan et Evermann, 1896) & Control region & 7.87 & Bernardi et al. 2003 \\
\hline \multirow[t]{2}{*}{ Hypsopsetta guttulata (Girard, 1856) } & Control region & 0.1211 & Schinske et al. 2010 \\
\hline & S7 & 0.0029 & Schinske et al. 2010 \\
\hline Lythrypnus dalli (Gilbert, 1890) & Cytochrome B & 0.20 & Bernardi et al. 2003 \\
\hline Myliobatis californica Gill, 1865 & NADH2 & 0.3 & SCRO \\
\hline Narcine entemedor Jordan et Starks, 1895 & NADH2 & 0 & SCRO \\
\hline Paralabrax maculatofasciatus (Steindachner, 1868) & Control region & 1.06 & Stepien et al. 2001 \\
\hline \multirow[t]{2}{*}{ Rhinobatos productus Ayres, 1854} & Control region & 2.47 & SCRO \\
\hline & $\mathrm{NADH} 2$ & 1.2 & SCRO \\
\hline Rhinoptera steindachneri Evermann et Jenkins, 1891 & NADH2 & 10 & $\begin{array}{l}\text { Sandoval-Castillo and } \\
\text { Rocha-Olivares } 2011\end{array}$ \\
\hline Sebastes macdonaldi (Eigenmann et Beeson, 1893) & Control region & 0.64 & Bernardi et al. 2003 \\
\hline Semicossyphus pulcher (Ayres, 1854) & Control region & 0.84 & Bernardi et al. 2003 \\
\hline
\end{tabular}

SCRO = Sandoval-Castillo and Rocha-Olivares, unpublished data in: Sandoval-Castillo and Rocha-Olivares (2011).

McEachran et Miyake, 1988 (Rajidae), Urobatis concentricus Osburn et Nichols, 1916 (Urotrygonidae), Urolophus maculatus (Garman, 1913) (Urolophidae), Gymnothorax eurygnathos Böhlke, 2001 (Muraenidae), Herpetoichthys fossatus (Myers et Wade, 1941) (Ophichthidae), Heteroconger digueti (Pellegrin, 1923) (Congridae), Anchoa mundeoloides (Breder, 1928) (Engraulidae), Porichthys analis Hubbs et Schultz, 1939 (Batrachoididae), Scorpaena sonorae Jenkins et Evermann, 1889 (Scorpaenidae), Mycteroperca prionura Rosenblatt et Zahuranec, 1967, M. rosacea (Streets, 1877) (Serranidae), Opistognathus rosenblatti Allen et Robertson, 1991 (Opistognathidae), Orthopristis reddingi Jordan et Richardson, 1895 (Haemulidae), Chromis limbaughi Greenfield et Woods, 1980, Stegastes rectifraenum (Gill, 1862) (Pomacentridae), Enneanectes reticulatus Allen et Robertson, 1991 (Tripterygiidae), Labrisomus xanti Gill, 1860 (Labrisomidae), Cirriemblemaria lucasana (Stephens, 1963) (Chaenopsidae), Bollmannia macropoma Gilbert, 1892, B. ocellata Gilbert, 1892 (Gobiidae), and Citharichthys gordae Beebe et Tee-Van, 1938 (Paralichthyidae). The record of Paralichthys aestuarius from Laguna Ojo de Liebre (Arellano-Martinez et al. 1997) is erroneous; this specimen was examined and allowed us to confidently identify it as P. californicus (Ayres, 1859).

On the other hand, ecological characteristics of the CP endemic species revealed that the component associated with coralline and rocky reef systems is dominant, mainly represented by small fishes from the families Gobiidae,
Chaenopsidae, Labrisomidae, Gobiesocidae, and Bythitidae, among others. These groups are common off the islands of the Gulf of California, and in the central and south-western coasts of it (Thomson et al. 2000, Thomson and Gilligan 2002). Demersal, demersal-pelagic, and neritic-pelagic species from the families Paralichthyidae, Sciaenidae, Engraulidae, and Atherinopsidae are related mainly to soft bottom ecosystems in the eastern coast and Upper Gulf of California (Hastings and Findley 2007, Robertson and Allen 2008). Concerning deep-water ichthyofauna from the Gulf of California, Castro-Aguirre and Balart (1996) denoted the importance of further studies on the great basins, trenches, and ocean depressions from where specimens like the Cortez hagfish, Eptatretus sinus, were obtained. Robertson and Cramer (2009) without counting this deep-water component validated the existence of only 62 fish species endemic to the PC.

From the endemic ichthyofauna, 12 species reach a maximum length of $5 \mathrm{~cm}, 39$ are shorter than $10 \mathrm{~cm}$, and only the totoaba reaches a total length exceeding $2 \mathrm{~m}$. Small-sized species belong mainly to the families Gobiidae, Chaenopsidae, Labrisomidae, Gobiesocidae, Dactyloscopidae, and Tripterygiidae. The dominant reproduction strategy of these families is oviparous with benthic eggs and pelagic phase. These families are considered primary residents given the limited movility of larvae and adults (Thomson and Gilligan 2002). Eggs are relatively large and fixed to the substratum, while adults have a short lifetime (one or two years of generation time), becoming 
territorial and therefore limiting genetic flow and favouring species fragmentation (Rosenblatt 1963, Thomson and Gilligan 2002). TEP biogeographic gaps (e.g., Sinaloa and Central America) have a considerable impact on these families. Conversely oviparous species with pelagic eggs and long planktonic larval stage (secondary residents) have great potential for dispersal, which is enhanced by the currents (Leis and McCormick 2002), keeping species genetic homogeneity. In the TEP just $30 \%$ of species are oviparous with benthic eggs and pelagic phase, most of the species are oviparous with pelagic eggs (56\%) (Robertson and Allen 2008).

Some models have demonstrated that ecological differences may cause partial or total reproductive barriers in just hundreds of generations (Hendry et al. 2007). Reproductive isolation may evolve rapidly when populations are settled and adapted to ecologically different environments (Palumbi 1994). In the presently reported study, 26 species with disjunct population distribution have been detected; these species are found on the Pacific Coast and in the northern part of the Gulf of California, but are absent from the southern (Cabo San Lucas) region (Walker 1960, Castro-Aguirre et al. 2005a, Hastings et al. 2010). These species are: Hydrolagus colliei (Lay et Bennett, 1839) (Chimaeridae), Raja binoculata Girard, 1855, R. inornata Jordan et Gilbert, 1881, R. rhina Jordan et Gilbert, 1880 (Rajidae), Platyrhinoidis triseriata (Jordan et Gilbert, 1880) (Platyrhinidae), Atherinops affinis (Ayres, 1860) (Atherinopsidae), Scorpaena guttata Girard, 1854 (Scorpaenidae), Sebastes macdonaldi (Sebastidae), Zaniolepis frenata Eigenmann et Eigenmann, 1889 (Hexagrammidae), Anisotremus davidsonii (Haemulidae), Atractoscion nobilis (Ayres, 1860), Cheilotrema saturnum (Girard, 1858) (Sciaenidae), Girella nigricans (Kyphosidae), Zalembius rosaceus (Jordan et Gilbert, 1880) (Embiotocidae), Halichoeres semicinctus, Semicossyphus pulcher (Labridae), Hypsoblennius gentilis (Girard, 1854), H. jenkinsi (Blenniidae), Exerpes asper (Jenkins et Evermann, 1889) (Labrisomidae), Chaenopsis alepidota (Chaenopsidae), Gillichthys mirabilis (Gobiidae), Scomberomorus concolor (Lockington, 1879) (Scombridae), Paralichthys californicus (Paralichthyidae), Pleuronichthys guttulatus (Girard, 1856), P. ocellatus Starks et Thompson, 1910, and P. verticalis Jordan et Gilbert, 1880 (Pleuronectidae).

Some studies (e.g., Bernardi et al. 2003, SandovalCastillo and Rocha-Olivares 2011) have detected subtle morphological differences and, in some species, significant genetic distance (Table 2), which in turn could be derived in increasing ichthyodiversity endemism in this province. In addition, according to Robertson and Allen (2008), three species (Chriolepis (2) and Enneanectes (1)) have not yet been described in the Gulf of California.

There is a general consensus that the limits of biogeographic provinces are identified by the occurrence of species distribution and that these limits generally correspond with the existence of environmental discontinuities. Established limits for the CP have been extensively discussed (e.g.,
Hendrickx 1992, Hastings 2000, Erisman et al. 2011). Based on geological evidence several authors (Thomson et al. 2000, Brusca and Findley 2005, Brusca et al. 2005, Hendrickx et al. 2005, 2007, and Hastings et al. 2010) have included in the limits of the Gulf of California an extended fringe from the Baja California Peninsula towards the Mexican mainland territory, in other words from Cabo San Lucas, BCS towards Cabo Corriente, Jalisco. The southern CP limit extension allows the inclusion of three endemic species from Islas Marías: the scaly-belly blenny, Starksia lepidogaster Rosenblatt et Taylor, 1971, described from 11 specimens collected at Cleopatra Island; the leastfoot blenny, Paraclinus ditrichus Rosenblatt et Parr, 1969, known only from three specimens; and the lonely clingfish, Gobiesox marijeanae Briggs, 1960, known from eleven specimens. The latter two species were sampled at San Juanito Island. This addendum would increase endemic species number to 82 .

A great number of studies have pointed out the importance of the CP in terms of conservation, and although recent meaningful efforts for its conservation have been developed (e.g., World heritage Islands and protected areas of the Gulf of California, Natural protected areas: Biosphere reserves of the Upper Gulf of California and Colorado River delta; San Pedro Martir Island; Bahia de los Angeles, Ballenas and Salsipuedes Channels; Gulf of California islands; and Cabo San Lucas; National parks of Loreto bay, Cabo Pulmo, Espiritu Santo Archipelago, and San Lorenzo Archipelago), over the past two decades the existence of a complex web of threats to this large marine ecosystem have being noted (Sala et al. 2004, Enríquez-Andrade et al. 2005, Sáenz-Arroyo et al. 2005, Cudney-Bueno et al. 2009, Hastings et al. 2010, Erisman et al. 2011).

It is generally known that nomenclature ambiguity, and incomplete diversity lists may represent a problem for species and therefore area conservation (Rojas 1992, Hey et al. 2003, Isaac et al. 2004, Mace 2004). Hence, the importance of clarifying species taxonomic status and updating the knowledge of endemism might help in the development of objective conservation strategies (Kerr 1997).

\section{ACKNOWLEDGEMENTS}

This work was funded by the IIK'KAKNAB, A.C. We thank to Alfredo Zayas, Norma Sánchez, Pablo Castro, Luis Mejía, Carlos Felix, and Linda Barranco for their contribution to this study. D.S.P.S. wishes to thank CONACyT's program: 'Complementary Support for the Institutional Strengthening Working Groups: Retention'. Thanks to Melba De Jesús Huerta and Mariana Santana for their help in language editing.

\section{REFERENCES}

Anonymous 1999. International code of zoological nomenclature. Fourth edition. International Commission of Zoological Nomenclature. The International Trust for Zoological Nomenclature, c/o Natural History Museum, London.

Aragón-Noriega E.A., Valenzuela-Quiñones W., EsparzaLeal H., Ortega-Rubio A., Rodríguez-Quiroz G. 2009. 
Analysis of management options for artisanal fishing of the Bigeye Croaker Micropogonias megalops (Gilbert, 1890) in the Upper Gulf of California. International Journal of Biodiversity Science and Management 5 (4): 208-214. DOI: $10.1080 / 17451591003709371$

Arellano-Martínez M., De La Cruz-Agüero J., Cota-Gómez V.M. 1997. New records and range extensions of fishes on the Pacific Coast of Mexico. Revista Biología Tropical 45 (2): 936.

Balon E.K. 1989. Patterns in the evolution of reproductive styles in fishes. Pp. 35-51. In: Potts G.W., Wootton R.J. (eds.). Fish reproduction strategies and tactics. Academic Press, London.

Bath H. 2008. Review of the genus Parablennius Miranda-Ribeiro from Australia and New Caledonia (Pisces: Blenniidae: Salariinae). Stuttgarter Beiträge zur Naturkunde A, Neue Serie 1: 77-94.

Berdegue J. 1955. La pesquería de la totoaba (Cynoscion macdonaldi Gilbert) en San Felipe, Baja California. Revista de la Sociedad Mexicana de Historia Natural 16 (1-4): 45-78.

Bernardi G., Findley L.T., Rocha-Olivares A. 2003. Vicariance and dispersal across Baja California in disjunct marine fish populations. Evolution 57 (7): 1599-1609.

Bernardi G., Lape J. 2005. Tempo and mode of speciation in the Baja California disjunct fish species Anisotremus davidsonii. Molecular Ecology 14 (13): 4085-4096. DOI: 10.1111/j.1365-294X.2005.02729.x

Briggs J.C. 1974. Marine zoogeography. McGraw-Hill, New York, NY, USA.

Brusca R.C., Findley L.T. 2005. El Mar de Cortés. Pp: 1-32. In: Hendrickx M.E., Brusca C.R., Findley L.T. (eds.) Listado y Distribución de la Macrofauna del Golfo de California, México. Part I. Invertebrados. Arizona-Sonora Desert Museum/Conservation International, Tucson, AZ, USA.

Brusca R.C., Findley L.T., Hastings P.A., Hendrickx M.E., Torre Cosio J., van der Heiden A.M. 2005. Macrofaunal biodiversity in the Gulf of California. Pp. 179-203. In: Cartron J.L.E., Ceballos G., Felger R. (eds.) Biodiversity, ecosystems, and conservation in Northern Mexico. Oxford University Press, New York, NY, USA.

Bussing W.A., Lavenberg R.J. 2003. Four new species of eastern tropical Pacific jawfishes (Opistognathus: Opistognathidae). Revista Biología Tropical 51 (2): 529-550.

Castro-Aguirre J.L., Antuna-Mendiola A., González-Acosta A.F., De la Cruz-Agüero J. 2005a. Mustelus albipinnis sp. nov. (Chondrichthyes: Carcharhiniformes: Triakidae) de la costa suroccidental de Baja California Sur, México. Hidrobiológica 15 (2): 123-130.

Castro-Aguirre J.L., Balart E.F. 1996. Contribución al conocimiento del origen y las relaciones de la ictiofauna de aguas profundas del Golfo de California, México. Hidrobiológica 6 (1-2): 67-76.

Castro-Aguirre J.L., Balart E.F., Arvizu-Martínez J. 1995. Contribución al conocimiento del origen y distribución de la ictiofauna del Golfo de California, Mexico. Hidrobiológica 5 (1-2): 57-78.

Castro-Aguirre J.L., Espinosa-Pérez H. 1996. Listados faunísticos de México. VII. Catalogo sistemático de las rayas y especies afines de México (Chondrichthyes: Elasmobranchii: Rajiformes: Batoideiomorpha). Instituto de Biología, UNAM, Mexico.

Castro-Aguirre J.L., Espinosa-Pérez H. 2006. Los peces de la familia Atherinopsidae (Teleostei: Atheriniformes) de las lagunas costeras neutras e hipersalinas de México. Hidrobiológica 16 (1): 89-101.

Castro-Aguirre J.L., Gonzalez-Acosta A.F., De la CruzAgüero J. 2005b. Lista anotada de las especies ícticas anfipacificas, de afinidad boreal, endémicas y anfipeninsulares del Golfo de California, México. Universidad y Ciencia 21 (42): 85-106.

Castro-Aguirre J.L., González-Acosta A.F., De la CruzAgüero J., Moncayo-Estrada R. 2006. Ictiofauna marinacostera del Pacífico central mexicano: análisis preliminar de su riqueza y relaciones biogeográficas. Pp: 149-166. In: Jiménez-Quiroz M.C., Espino-Barr E. (eds.) Los recursos pesqueros y acuícolas de Jalisco, Colima y Michoacán. Instituto Nacional de Pesca, SAGARPA, Mexico.

Chen L.C. 1975. The rockfishes, genus Sebastes (Scorpaenidae), of the Gulf of California, including three new species with a discussion of their origin. Proceedings of the California Academia of Sciences Ser. 4, 40 (6): 109-141.

Cisneros-Mata M.A., Montemayor-López G., RománRodríguez M.J. 1995. Life history and conservation of Totoaba macdonaldi. Conservation Biology 9 (4): 806-814. DOI: 10.1046/j.1523-1739.1995.09040806.x

Compagno L.J.V. 2005. Checklist of living Elasmobranchs. Pp. 503-548. In: Hamlett W.C. (ed.) Reproductive biology and phylogeny of Chondrichthyes: sharks, batoids, and chimaeras. Science Publishers, Enfield, NH, USA.

Cudney-Bueno R., Bourillón L., Sáenz-Arroyo A., TorreCosío J., Turk-Boyer P., Shaw W.W. 2009. Governance and effects of marine reserves in the Gulf of California, Mexico. Ocean and Coastal Management 52 (3-4): 207-218. DOI: 10.1016/j.ocecoaman.2008.12.005

Dawson C.E. 1975. Studies on the eastern Pacific sand stargazers (Pisces: Dactyloscopidae) 2. Genus Dactyloscopus, with descriptions of new species and subspecies. Natural History Museum of Los Angeles County, Scientific Bulletin 22 (1): 1-61.

Elliott M., Dewailly F. 1995. The structure and components of European estuarine fish assemblages. Netherlands Journal of Aquatic Ecology 29 (3-4): 397-417.

DOI: $10.1007 / \mathrm{BF} 02084239$

Enríquez-Andrade R., Anaya-Reyna G., Barrera-Guevara J.C., Carvajal-Moreno M.A., Martínez-Delgado M.E., VacaRodríguez J., Valdés-Casillas C. 2005. An analysis of critical areas for biodiversity conservation in the Gulf of California region. Ocean and Coastal Management 48 (1): $31-50$.

DOI: 10.1016/j.ocecoaman.2004.11.002

Erisman B.E., Paredes G.A., Plomozo-Lugo T., Cota-Nieto J.J., Hastings P.A., Aburto-Oropeza O. 2011. Spatial structure of commercial marine fisheries in Northwest Mexico. ICES Journal of Marine Science 68 (3): 564-571.

DOI: $10.1093 /$ icesjms/fsq179

Findley L.T., Hastings P.A., van der Heiden A.M., Güereca R., Torre J., Thomson D.A. 1997. Distribution of endemic 
fishes of the Gulf of California, Mexico. Pp. 130. In: Abstracts, 77th Annual Meeting of the American Society of Ichthyologists and Herpetologists, Seattle, WA, USA.

Findley L.T., Hastings P.A., van der Heiden A.M., Güereca R., Torre J., Thomson D.A. 1999. Distribution of endemic ichthyofauna of the Sea of Cortes. Pp. 51. In: Abstracts, VII Congreso de la Asociación de Investigadores del Mar de Cortés, A.C. and Simposium Internacional sobre el Mar de Cortés. 25-28, Mayo 1999. Universidad de Sonora. Hermosillo, Sonora, Mexico.

Findley L.T., Torre J., Hastings P.A., van der Heiden A.M., Nava J.M. 1996a. Diversity and endemicity of the fish fauna of the Gulf of California, Mexico. Pp. 10-13. In: Program and Abstracts. The phylogeny of life and the accomplishments of phylogenetic biology. A symposium at the University of Arizona Tucson, AZ, USA.

Findley L.T., Torre J., Nava J.M., van der Heiden A.M., Hastings P.A. 1996b. Preliminary ichthyofaunal analysis from a macrofaunal database on the Gulf of California, México. P. 138. In: Abstracts, 76th Annual Meeting of the American Society of Ichthyologists and Herpetologists, New Orleans, LA, USA.

Froese R., Pauly D. (eds.) 2012. FishBase. [version 06/2012] http://www.fishbase.org.

Hastings P.A. 2000. Biogeography of the Tropical Eastern Pacific: distribution and phylogeny of chaenopsid fishes. Zoological Journal of the Linnean Society 128 (3): 319-335. DOI: 10.1006/zjls.1998.0196

Hastings P.A., Findley L.T. 2007. Marine fishes of the Biosphere Reserve, Northern Gulf of California. Pp. 364-382. In: Felger R., Broyles W. (eds.) Dry borders: Great natural areas of the Gran Desierto and Upper Gulf of California. University Utah Press, Salt Lake City, UT, USA.

Hastings P.A., Findley L.T., Van der Heiden A.M. 2010. Fishes of the Gulf of California. Pp: 96-118. In: Brusca R. (ed.). The Gulf of California. Biodiversity and Conservation. University Arizona Press. Tucson, AZ, USA.

Hastings P.A., Springer V.G. 2009. Recognizing diversity in blennioid fish nomenclature (Teleostei: Blennioidei). Zootaxa 2009 (2120): 3-14.

Hendrickx M.E. 1992 Distribution and zoogeographic affinities of decapods crustaceans of the Gulf of California, Mexico. Proceedings of the San Diego Society of Natural History 20 (1): 1-11.

Hendrickx M.E., Brusca R.C., Cordero M., Ramírez R.G. 2007. Marine and brackish-water molluscan biodiversity in the Gulf of California, Mexico. Scientia Marina 71 (4): 637-647. DOI: $10.3989 /$ scimar.2007.71n4637

Hendrickx M.E., Brusca R.C., Findley L.T. (eds.) 2005. A Distributional checklist of the macrofauna of the Gulf of California, Mexico. Part I. Invertebrates. Arizona-Sonora Desert Museum and Conservation International.

Hendry A.P., Nosil P., Rieseberg L.H. 2007. The speed of ecological speciation. Functional Ecology 21 (3): 455-464. DOI: 10.1111/j.1365-2435.2007.01240.x

Hey J., Waples R.S., Arnold M.L., Butlin R.K., Harrison R.G. 2003. Understanding and confronting species uncertainty in biology and conservation. Trends in Ecology and Evolution 18 (11): 597-603.

DOI: $10.1016 /$ j.tree.2003.08.014
Hildebrand S.F. 1943. A review of the American anchovies (family Engraulidae). Bulletin of the Bingham Oceanographic Collection Yale University 8: 1-165.

Huang D., Bernardi G. 2001. Disjunct Sea of Cortez-Pacific Ocean Gillichthys mirabilis populations and the evolutionary origin of their Sea of Cortez endemic relative, Gillichthys seta. Marine Biology 138 (2): 421-428. DOI: $10.1007 / \mathrm{s} 002270000454$

Isaac N.J.B., Mallet J., Mace G.M. 2004. Taxonomic inflation: its influence on macroecology and conservation. Trends in Ecology and Evolution 19 (9): 464-469. DOI: $10.1016 /$ j.tree.2004.06.004

Kerr J.T. 1997. Species richness, endemism, and the choice of areas for conservation. Conservation Biology 11 (5): 1094-1100. DOI: $10.1046 / \mathrm{j} .1523-1739.1997 .96089 . x$

Leis J.M., McCormick M.I. 2002. The biology, behavior, and ecology of the pelagic, larval stage of coral reef fishes. Pp 171-199. In: Sale P.F. (ed.) Coral reef fishes dynamics and diversity in a complex ecosystem. Academic Press, London.

Love M.S., Yoklavich M., Thorsteinson L. 2002. The rockfishes of the Northeast Pacific. University of California Press, Berkeley, CA, USA.

Mace G.M. 2004. The role of taxonomy in species conservation. Philosophical Transactions of the Royal Society of London B 359 (1444): 711-719.

DOI: $10.1098 /$ rstb.2003.1454

Mora C., Robertson D.R. 2005. Factors shaping the range-size frequency distribution of the endemic fish fauna of the Tropical Eastern Pacific. Journal of Biogeography 32 (2): 277-286. DOI: $10.1111 / \mathrm{j} .1365-2699.2004 .01155 . \mathrm{x}$

Nelson J.S. 2006. Fishes of the world. 4th edn. John Wiley and Sons, Hoboken, NJ, USA.

Palumbi S.R. 1994. Genetic divergence, reproductive isolation, and marine speciation. Annual Review of Ecology and Systematics 25: 547-572. DOI: 10.1146/annurev.es.25.110194.002555.

Paredes G.A., Erisman B., Mascareñas-Osorio I., Cota-Nieto J., Gherard K., Aburto-Oropeza O. 2010. La curvina golfina: Biologia, pesqueria y su gente. CONABIO. Biodiversitas 2010 (91): 1-5.

Pérez Jiménez J.C., Sosa Nishizaki O., Castillo Geniz J.L. 2005. A new eastern north Pacific smoothhound shark (genus Mustelus, family Triakidae) from the Gulf of California. Copeia 2005 (4): 834-845.

DOI: 10.1643/0045-8511(2005)005[0834:ANENPS]2.0.CO;2

Robertson D.R., Cramer K.L. 2009. Shore fishes and biogeographic subdivisions of the tropical eastern Pacific. Marine Ecology Progress Series 380: 1-17.

DOI: $10.3354 /$ meps 07925

Robertson D.R., Allen G.R. 2008. Peces costeros del Pacífico oriental tropical: Sistema de informacion en linea. Version 1.0 (2008). Instituto Smithsonian de Investigaciones Tropicales, Balboa, Republica de Panama. www.neotropicalfishes.org/sftep.

Robertson D.R., Grove J.S., McCosker J.E. 2004. Tropical transpacific shore fishes. Pacific Science 58 (4): 507-565. DOI: $10.1353 /$ psc.2004.0041 
Rojas M. 1992. The species problem and conservation: What are we protecting? Conservation Biology 6 (2): 170-178. DOI: $10.1046 / \mathrm{j} .1523-1739.1992 .620170 . x$

Rosenblatt R.H. 1963. Some aspects of speciation in marine shore fishes. Pp. 171-180. In: Harding J.P., Tebble N.B. (eds.) Speciation in the sea. Publications of the Systematics Association, London, No. 5.

Rosenblatt R.H, Parr T.D. 1967. The identity of the blenny Paraclinus altivelis (Lockington) and the status of $P$. sinus Hubbs. Copeia 1967 (3): 675-677.

Rosenblatt R.H., Parr T.D. 1969. The Pacific species of the clinid fish genus Paraclinus. Copeia 1969 (1): 1-20.

Sáenz-Arroyo A., Roberts C.M., Torre J., Cariño-Olvera M., Enríquez-Andrade R.R. 2005. Rapidly shifting environmental baselines among fishers of the Gulf of California. Proceedings of the Royal Society of London B 272 (1575): 1957-1962.

DOI: $10.1098 / \mathrm{rspb} .2005 .3175$

Sala E., Aburto-Oropeza O., Reza M., Paredes G., LópezLemuse L.G. 2004. Fishing down coastal food webs in the Gulf of California. Fisheries 29 (3): 19-25. DOI: 10.1577/1548-8446(2004)29[19:fdcfwi]2.0.co;2

Sandoval-Castillo J.R., Rocha-Olivares A. 2011. Deep mitochondrial divergence in Baja California populations of an aquilopelagic elasmobranch: the golden cownose ray. Journal of Heredity 102 (3): 269-274.

DOI: $10.1093 /$ jhered/esr004

Schinske J.N., Bernardi G., Jacobs D.K., Routman E.J. 2010. Phylogeography of the diamond turbot (Hypsopsetta guttulata) across the Baja California Peninsula. Marine Biology 157 (1): 123-134.

DOI: $10.1007 / \mathrm{s} 00227-009-1302-2$

Springer V.G. 1959. Systematics and zoogeography of the clinid fishes of the subtribe Labrisomini Hubbs. Publications of the Institute of Marine Science, University of Texas 5: 417-492.

Stepien C., Rosenblatt R.H. 1991. Patterns of gene flow and genetic divergence in the northeastern Pacific Clinidae (Teleostei: Blennioidei), based on allozyme and morphological data. Copeia 1991 (4): 873-896.

DOI: $10.2307 / 1446084$
Stepien C.A., Rosenblatt R.H., Bargmeyer B.A. 2001. Phylogeography of the spotted sand bass, Paralabrax maculatofasciatus: divergence of Gulf of California and Pacific coast populations. Evolution 55 (9): 1852-1862. DOI: 10.1111/j.0014-3820.2001.tb00834.x

Swift C.C., Findley L.T., Ellingson R.A., Flessa K.W., Jacobs D.K. 2011. The delta mudsucker, Gillichthys detrusus, a valid species (Teleostei: Gobiidae) endemic to the Colorado River delta, northernmost Gulf of California, México. Copeia 2011 (1): 93-102. DOI: $10.1643 / \mathrm{CI}-09-123$

Terry A., Bucciarelli G., Bernardi G. 2000. Restricted gene flow and incipient speciation in disjunct Pacific Ocean and Sea of Cortez populations of a reef fish species, Girella nigricans. Evolution 54 (2): 652-659.

DOI: 10.1111/j.0014-3820.2000.tb00066.x

Thomson D.A., Findley L.T., Kerstitch A.N. 2000. Reef Fishes of the Sea of Cortez: The Rocky-Shore Fishes of the Gulf of California. University of Texas Press, Austin, TX, USA.

Thomson D.A., Gilligan M. 2002. Rocky-shore fishes. Pp: 154-180. In: Case T.J., Cody M.L., Ezcurra E. (eds.) A new island biogeography in the Sea of Cortes. Oxford University Press, Oxford.

Walker B.W. 1960. The distribution and affinities of the marine fish fauna of the Gulf of California. Symposium: The Biogeography of Baja California and adjacent Seas. System Zoology 9 (3): 123-133.

Whitehead P.J.P., Nelson G.J., Wongratana T. 1988. FAO Species Catalogue. Clupeoid fishes of the world (Suborder Clupeoidei). An annotated and illustrated catalogue of the herrings, sardines, pilchards, sprats, shads, anchovies and wolfherrings. Vol. 7. Part 2. Engraulididae. FAO Fish Synopsis 125 .

Received: 5 May 2012

Accepted: 22 June 2012

Published electronically: 30 September 2012 